\title{
A Comparative Analysis of Thresholding and Edge Detection Segmentation Techniques
}

\author{
Jaskirat Kaur \\ Student UIET \\ Chandigarh
}

\author{
Sunil Agrawal \\ Assistant prof.,UIET \\ Panjab university \\ Chandigarh
}

\author{
Renu Vig \\ Director, UIET \\ Panjab university \\ Chandigarh
}

\begin{abstract}
Thresholding and edge detection being one of the important aspects of image segmentation comes prior to feature extraction and image recognition system for analyzing images. It helps in extracting the basic shape of an image, overlooking the minute unnecessary details. In this paper using image segmentation (thresholding and edge detection) techniques different geo satellite images, medical images and architectural images are analyzed. To quantify the consistency of our results error measure is used.
\end{abstract}

\section{General Terms}

Image segmentation, edge detection, thresholding

\section{Keywords}

$e_{r m s}$, Error measures, algorithms

\section{INTRODUCTION}

Image segmentation techniques play an important role in image recognition system. It helps in refining our study of images. One part being edge and line detection techniques highlights the boundaries and the outlines of the image by suppressing the background information. They are used to study adjacent regions by separating them from the boundary.

The main problem of quantifying different edge detectors is that there is no unique way of studying an image. For example, each person has its own perception of segmenting and analyzing the image. If we reach at two different results arising from the same image then one single particular technique will be declared inconsistent [1].

This paper presents a quantitative study of different edge detection and thresholding techniques. Comparison is done on images from medical, geo spatial and architectural fields. Edge detectors like prewitt, canny and sobel were implemented and tested on various images from these fields. The characteristic used to measure the consistency of these edge detectors is $e_{r m s}$, which is root mean square error between the input image and the output image. This measure allows a principled comparison between different results generated by the edge detectors.

This paper is organized a follows: section 2 related work is briefly presented. Section 3 describes the image segmentation and the edge detectors used. Section 4 presents the experimental results. Section 5 concludes the paper.

\section{RELATED WORK}

There is a huge literature on segmentation dating back to decades, with applications in myriad areas. In this section, some of the related work is presented relevant to the approach of this paper. In classical threshold image segmentation [2] an image is segmented and simply sorted to object and background by setting a threshold. It is easy to get good results by threshold segmentation. However, if there is complex information in an image, the threshold algorithm is definitely not suitable. Edge detectors have been evaluated based on average risk [9]. It is the performance measure based on Bayesian decision theory. In this performance, edge detector is context dependent which makes it non-trivial. Edges are detected for the images in spatial domain and edge detectors are evaluated based on the relative frequencies of the edge detected pixels and edge differences.

\section{IMAGE SEGMENTATION}

Image Segmentation is the process of dividing a digital image into constituent regions or objects [4]. The purpose of segmentation is to simplify the representation of an image into that which is easier to analyze. Image segmentation is typically used to locate objects and boundaries in images. Segmentation algorithms are based on the two basic properties of an image intensity values: discontinuity and similarity. To study discontinuities in an image we divide image based on the abrupt changes in intensity, such as edges.

\subsection{Edge Detectors}

Edge detection is the most common approach for detecting meaningful discontinuities in intensity values. The edge is a boundary between two regions with relatively distinct gray level properties. Such discontinuities are detected by using first and second order derivatives. The first-order derivative in image processing is the gradient defined as below. The gradient of an image $f(x, y)$ at location $(x, y)$ is the vector[3]:

$\nabla f=\left[\frac{G_{x}}{G_{y}}\right]=\left[\frac{\partial f / \partial x}{\partial f / \partial y}\right]$

The gradient vector points in the direction of maximum rate of change of $f$ at $(x, y)$. In edge detection, an important parameter is the magnitude of this vector.

$$
|\nabla f|=\sqrt{G_{x}^{2}+G_{y}^{2}}
$$

The gradient takes it's maximum rate of increase of $f(x, y)$ per unit distance in the direction of $f$. To simplify computation, ignoring square root approximates this quantity.

$$
|\nabla f|=\left|G_{x}\right|+\left|G_{y}\right|
$$

The gradient vector points in the direction of the maximum rate of change of $\nabla f$ at coordinates (x, y). The angle at which the maximum rate of change occurs is 


$$
\alpha(x, y)=\tan ^{-1}\left(\frac{G_{y}}{G_{x}}\right)
$$

The frequently used edge detection techniques in image segmentation are:

(1) Sobel Edge Detection,

(2) Prewitt Edge Detection and

(3) Canny Edge Detection.

(4) Roberts Edge Detection

The description of these methods is as follows.

\section{(1) Sobel Edge Detection}

Sobel edge detector uses the masks as shown in the figure below to digitally approximate the first order derivatives $\mathrm{G} x$ and $\mathrm{G} y[3]$.

$$
\begin{aligned}
\mathrm{G}= & {\left[G_{x}^{2}+G_{y}^{2}\right]^{1 / 2} } \\
= & \left\{\left[\left(z_{7}+2 z_{6}+z_{9}\right)-\left(z_{1}+2 z_{4}+z_{7}\right)\right]^{2}\right. \\
& \left.+\left[\left(z_{3}+2 z_{6}+z_{9}\right)-\left(z_{1}+2 z_{4}+z_{7}\right)\right]^{2}\right\}^{1 / 2}
\end{aligned}
$$

In its most common usage, the input to the operator is a gray scale image, as is the output. Pixel values at each point in the output represent the estimated absolute magnitude of the spatial gradient of the input image at that point.

\begin{tabular}{|l|l|l|}
\hline$z_{1}$ & $z_{2}$ & $z_{3}$ \\
\hline$z_{4}$ & $z_{5}$ & $z_{6}$ \\
\hline$z_{7}$ & $z_{8}$ & $z_{9}$ \\
\hline
\end{tabular}

$$
\begin{aligned}
& \begin{array}{|c|l|l|}
\hline-1 & -2 & -1 \\
\hline 0 & 0 & 0 \\
\hline 1 & 2 & 1 \\
\hline
\end{array} \\
& \mathrm{G}_{\mathrm{x}}=\left(z_{7}+z_{8}+z_{9}\right) \\
& \left(z_{1}+z_{2}+z_{3}\right)
\end{aligned}
$$

\begin{tabular}{|c|l|l|}
\hline-1 & 0 & 1 \\
\hline-2 & 0 & 2 \\
\hline-1 & 0 & 1 \\
\hline
\end{tabular}

Sobel mask

$$
\begin{aligned}
\mathrm{G}_{\mathrm{y}}= & \left(z_{3}+2 z_{6}+z_{9}\right)- \\
& \left(z_{1}+2 z_{4}+z_{7}\right)
\end{aligned}
$$

\section{(2) Prewitt Edge Detector}

The prewitt edge detector uses the masks shown in figure below to digitally approximate the first derivatives $\mathrm{G} x$ and $\mathrm{G} y$. The parameters of prewitt edge detector are similar to Sobel parameters. Though Prewitt edge detector is simpler to implement than the Sobel detector, but it tends to produce somewhat noisier results. The Prewitt operator measures two components namely the vertical edge component, which is calculated using kernel $\mathrm{Gx}$ and the horizontal edge component, which is calculated using kernel Gy. $|\mathrm{Gx}|+|\mathrm{Gy}|$ gives an indication of the intensity of the gradient in the current pixel.

\begin{tabular}{|l|l|l|}
\hline-1 & -1 & -1 \\
\hline 0 & 0 & 0 \\
\hline 1 & 1 & 1 \\
\hline
\end{tabular}

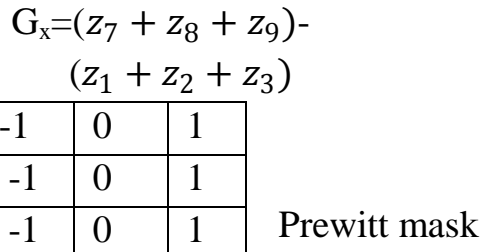

\begin{tabular}{|l|l|l|}
\multicolumn{1}{c}{} & $\left(z_{1}+z_{2}+z_{3}\right)$ \\
\hline-1 & 0 & 1 \\
\hline-1 & 0 & 1 \\
\hline-1 & 0 & 1 \\
\hline
\end{tabular}

$$
\mathrm{G}_{\mathrm{x}}=\left(z_{7}+z_{8}+z_{9}\right)-
$$$$
\left(z_{1}+z_{2}+z_{3}\right)
$$

\section{(3) Canny Edge Detector}

The canny edge detector is the most powerful edge detector. This technique finds edges by separating noise from the image before finding the edges of the image. It does not affect the features of the edges of the image. The method of finding edges using canny edge detector is as follows [7]:

(a) The image is smoothened using the Gaussian filter, to reduce noise.

(b) The local gradient, $\mathrm{G}(\mathrm{x}, \mathrm{y})=\left[G_{x}^{2}+G_{y}^{2}\right]^{1 / 2}$ and edge direction $\alpha(\mathrm{x}, \mathrm{y})=\tan ^{-1}\left(\mathrm{G}_{\mathrm{y}} / \mathrm{Gx}\right)$, are computed at each point. The advantage of using this method is that any of the Prewitt, Sobel, Roberts technique can be used to compute $\mathrm{G} x$ and Gy. Then edge point is a point whose strength is locally maximum in the direction of the gradient.

(c) The edge points detected in step 2 give rise to ridges in the gradient magnitude image. The algorithm then tracks along the top of these ridges and sets to zero all pixels that are not actually on the ridge top so as to give a thin line in the output, a process which is known as nonmaximal suppression. The ridge pixels are then thresholded using two thresholds, $\mathrm{T} 1$ and $\mathrm{T} 2$, with $\mathrm{T} 1<\mathrm{T} 2$. Ridge pixels with values greater than $\mathrm{T} 2$ are said to be strong edge pixels. Ridge pixels with values between $\mathrm{T} 1$ and $\mathrm{T} 2$ are said to be weak edge pixels.

(d) In the last step the algorithm performs edge linking by incorporating the weak pixels that are 8-connected to the strong pixels.

\section{(4) Roberts Edge Detection}

The Roberts edge detector computes 2-D spatial gradient on the image. It highlights regions of high spatial frequency, which corresponds to edges. Input to the operator is a gray scale image, as is the output. Pixel values at each point in the output represents the estimated absolute magnitude of the spatial gradient of the input image at that point [4]. The mask used by the Roberts edge detector used is shown in the figure below. 


\begin{tabular}{|l|l|}
\hline-1 & 0 \\
\hline 0 & 1 \\
\hline
\end{tabular}

\begin{tabular}{|l|l|}
\hline 0 & -1 \\
\hline 1 & 0 \\
\hline
\end{tabular}

\subsection{Thresholding}

Thresholding is one of the simplest image segmentation techniques. A threshold is chosen according to the application for which it is applied. If one particular image has light objects in the dark background, one way to extract the objects in the background is to select a threshold $\mathrm{T}$ that separates background and foreground objects [5]. The point for which $f(x, y) \geq \mathrm{T}$ is called an object point; otherwise the point is called as background point.

$$
g(x, y)=\left\{\begin{array}{l}
1 \text { if } f(x, y) \geq T \\
0 \text { if } f(x, y)<T
\end{array}\right\}
$$

There are numerous ways of selecting threshold like visual inspection, trial and error but these methods consume a lot of time .The best way for choosing threshold automatically is given in the following procedure [10]:

(a) Select an initial estimate for T. Generally it is the mid point between the minimum and maximum intensity values of the image.

(b) Segment the image using T. This will produce two groups of pixels: G1, consisting of all pixels with intensity values $\geq$ $\mathrm{T}$, and $\mathrm{G} 2$, consisting of pixels with values $<\mathrm{T}$.

(c) Then compute the average intensity values $\mu_{1}$ and $\mu_{2}$ for the pixels in regions $G_{1}$ and $G_{2}$.

(d) In the last step compute a new threshold value:

$\mathrm{T}=1 / 2\left(\mu_{1}+\mu_{2}\right)$

(e) Repeat the steps from $\mathrm{b}$ to $\mathrm{d}$ until the difference in $\mathrm{T}$ in successive iterations is smaller than a predefined parameter.

\subsection{Root Mean Square Error}

The Root mean square error $\left(e_{r m s}\right)$ is a measure, which calculates the average magnitude of the error. The equation for the $e_{r m s}$ is given below. The difference between the forecast and corresponding observed values are each squared and then averaged over the sample. Finally, the square root of the average is taken. Since the errors are squared before they are averaged, the $e_{r m s}$ gives a relatively high weight to large errors. The input image is represented as $f(\mathrm{x}, \mathrm{y})$, output image $\widetilde{f}(\mathrm{x}, \mathrm{y})$ and the error with $\mathrm{e}(\mathrm{x}, \mathrm{y})$.

$$
e(x, y)=\tilde{f}(x, y)-f(x, y)
$$

And the total error between two images is

$$
\sum_{x=0}^{M-1} \sum_{y=0}^{N-1}[\tilde{f}(x, y)-f(x, y)]
$$

The rms error $e_{r m s}$ between input and the output is given as

$$
e_{r m s}=1 / M N\left[\sum_{x=0}^{M-1} \sum_{y=0}^{N-1}[\tilde{f}(x, y)-f(x, y)]^{2}\right]^{1 / 2}
$$

\section{EXPERIMENTAL RESULTS}

In this section, the results are presented which are obtained by applying different edge detectors: prewitt, canny, sobel and thresholding segmentation techniques. The error measure calculated and compared in this section.

The experiments were made on number of images [11] and one from each field is shown over here and the results are compared.

Medical images:

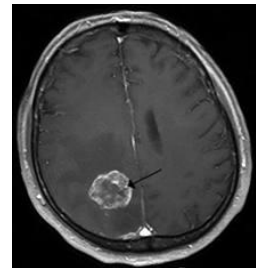

Original Image (Brain tumour)

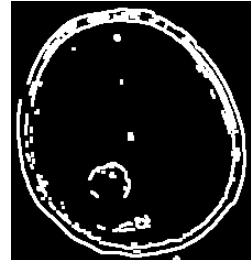

Segmentation using prewitt $e_{r m s}=292.5418$

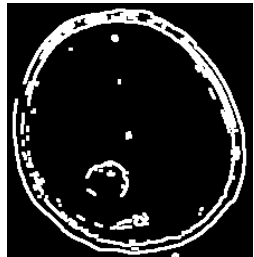

Segmentation using sobel $e_{r m s}=292.6503$

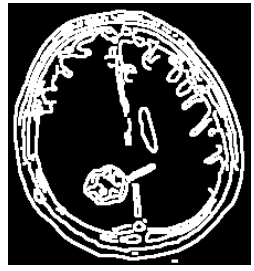

Segmentation using canny $e_{r m s}=292.0325$ (Best Result)

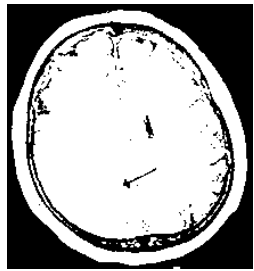

Segmentation using thresholding

$e_{r m s}=303.9847$
For brain tumours canny edge detector works best to detect tumours.

Figure 1.

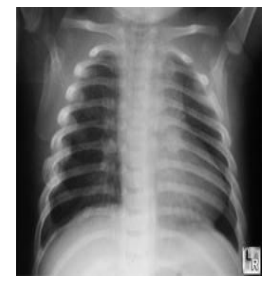

Original Image (lungs) 


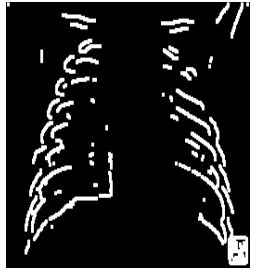

Segementation using prewitt

$$
e_{r m s}=245.5808
$$

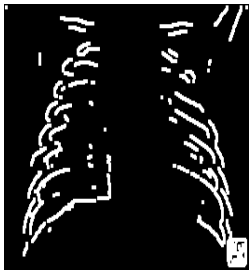

Segmentation using sobel

$e_{r m s}=245.5892$

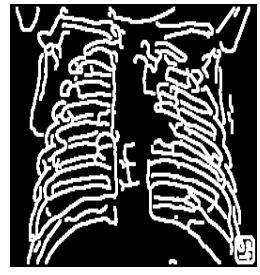

Segmentation using canny

$$
e_{r m s}=245.2815 \text { (Best }
$$
Result)

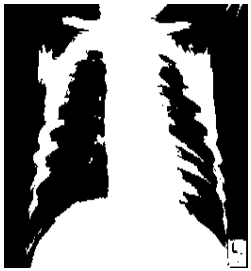

Segmentation using thresholding

$e_{r m s}=262.7583$

For fracture in the ribs canny edge detector provides the best amount of detail and least error.

Figure 2.

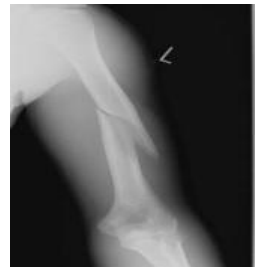

Original Image (fracture)

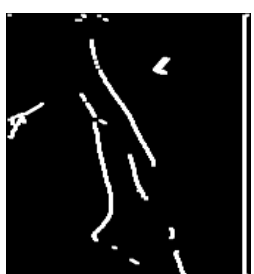

Segementation using prewitt $e_{r m s}=273.7957$

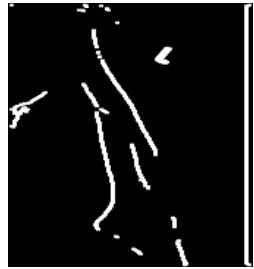

Segmentation using sobel $e_{r m s}=273.8051$

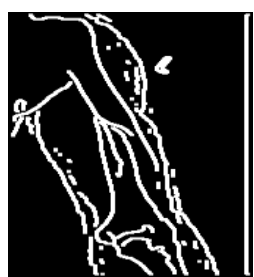

Segmentation using canny $e_{r m s}=273.7253$ (Best Results)

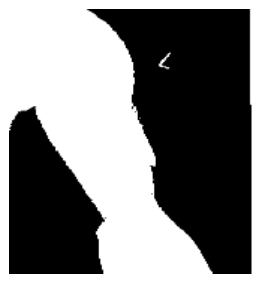

Segmentation using thresholding

$e_{r m s}=289.4375$

In the leg bone where actually the fracture is canny provides the detailed information with least error.

\section{Figure 3.}

Geo-spatial images:

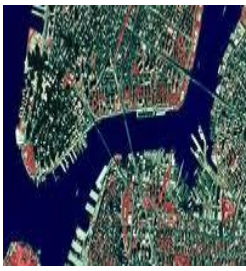

Original Image (geo-satellite)

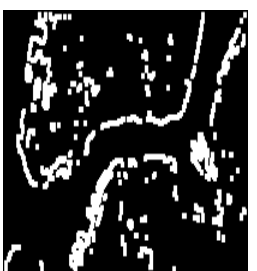

Segmentation using prewitt $e_{r m s}=279.8371$

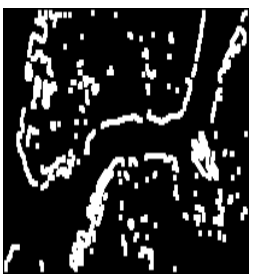

Segmentation using sobel $e_{r m s}=279.8456$

Another example of separating land and river going in between thresholding provides the best results

Figure 4.

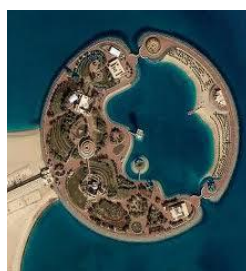

Original Image (remote sensing)

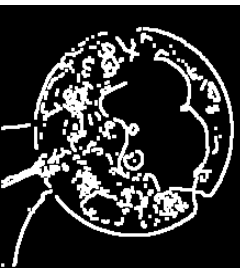

Segmentation using prewitt $e_{r m s}=276.2681$

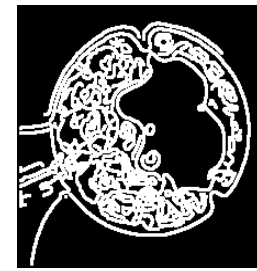

Segmentation using canny $e_{r m s}=276.2885$ 


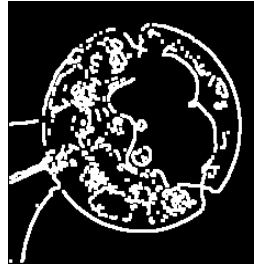

Segmentation using sobel

$$
e_{r m s}=276.2977
$$

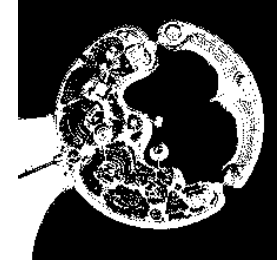

Segmentation using thresholding

$e_{r m s}=276.1223$ (Best results)
Clearly we can study the shape and separate water from the hotel area by thresholding.

Figure 5.

Architectural images:

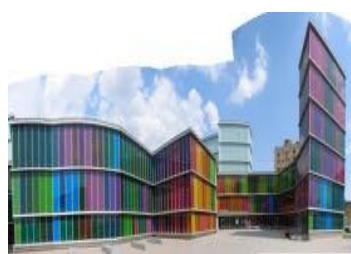

Original Image (architecture image)

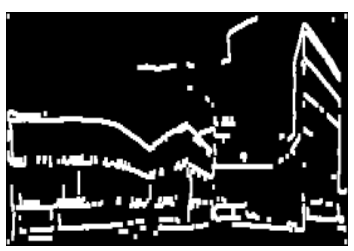

Segmentation using prewitt

$e_{r m s}=202.3350$ (Best results)

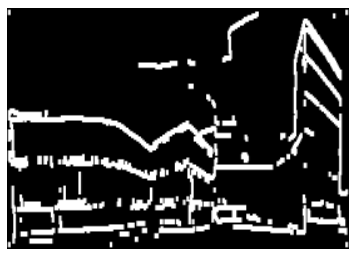

Segmentation using sobel

$e_{r m s}=202.3440$

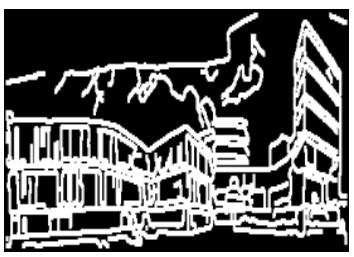

Segmentation using canny

$e_{r m s}=202.3453$

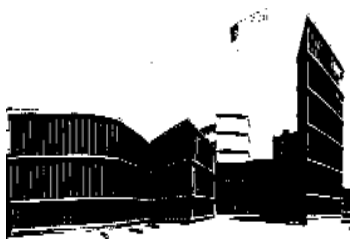

Segmentation using thresholding

$e_{\text {rms }}=202.3434$
To study the architecture of the building we need outlines, which are best provided by the prewitt edge detector.

Figure 6.

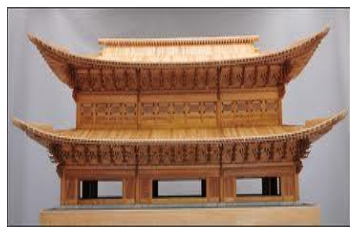

Original Image (traditional architecture image)

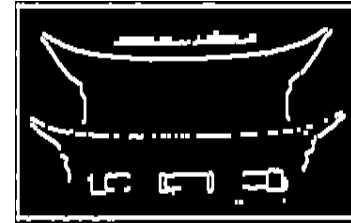

Segmentation using prewitt

$e_{r m s}=223.0636$ (Best results)

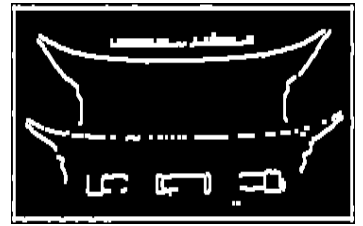

Segmentation using sobel

$$
e_{r m s}=223.0638
$$

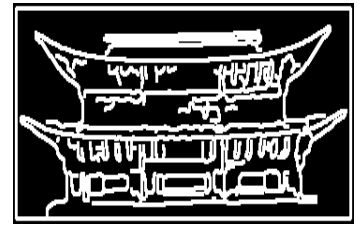

Segmentation using canny

$e_{r m s}=223.0832$

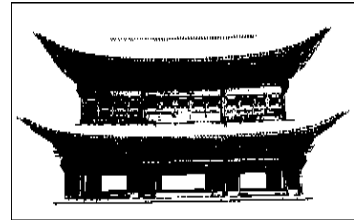

Segmentation using thresholding

$$
e_{r m s}=223.0953
$$

For the basic outline of the architecture we can either use prewitt or sobel with the minute difference in $e_{r m s}$.

Figure 7.

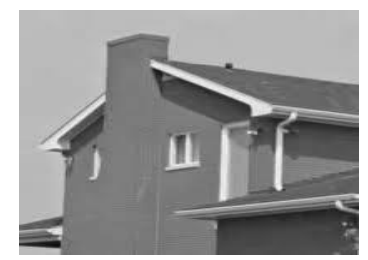

Original Image (house architecture image)

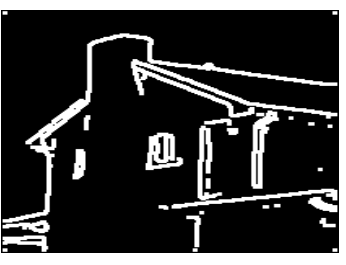

Segmentation using prewitt $e_{r m s}=212.1172$

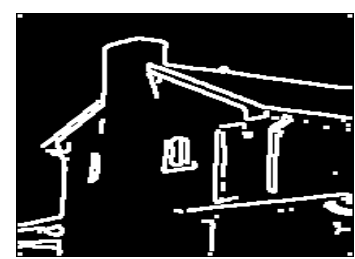

Segmentation using sobel

$e_{r m s}=212.1175$ (Best results)

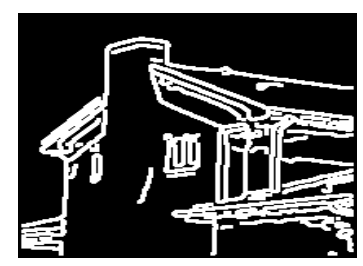

Segmentation using canny $e_{r m s}=212.9409$

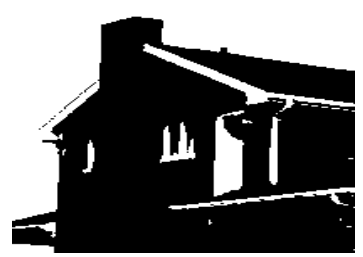

Segmentation using thresholding

$$
e_{\text {rms }}=212.9234
$$

Here also prewitt or sobel provides the almost equal results.

Figure 8.

The images taken over here were implemented over many other similar images to detect particular areas. Some of them as an example to the field are added in this section. Based on the application for the purpose we are analyzing the image is the idea behind getting the error between input and output. If that particular area is detected our purpose is solved and if not 
we need to use other detector which provides the best results with the least $e_{r m s}$ as shown in above tables.

\section{CONCLUSION}

This paper resulted a comparison of thresholding, different edge detection techniques on images from different fields. Observation was made that each type of image has different area to be analyzed. In this paper, images were examined using different techniques and the ones providing accurate results were identified. In the future work, the comparative study will be made by taking different clustering techniques used in image segmentation.

\section{REFERENCES}

[1] Alina Doringa, Gabriel Mihai, Dan Burdescu, "comparison of two image segmentation algorithms" Second international conference on advances in multimedia, IEEE computer society, pp. 185-190 (2010)

[2] $\mathrm{Mr}$ Salem Saleh Al-amri, Dr. N.V. Kalyankar, Dr.Khamitkar S.D "Image segmentation by using edge detection", International journal on computer science and engineering, vol. 02, No. 03, 2010, pp. 804-807

[3] N.Senthilkumaram, R.Rajesh "Edge detection techniques for image segmentation-A survey of soft computing approach", International journal of recent trends in engineering , vol.1, No.2, May 2009,pp. 250-254

[4] Steven L Eddins, Richard E.woods, Rafael C "digital image processing" (2009)
[5] Huang, Yourui; Wang, Shuang "Multilevel thresholding Methods for image segmentation with otsu based on QPSO”, Image and signal processing, CISP 2008, vol. 3,pp.701-705

[6] Shiping Zhu, Xi Xia, Qingrong Zhang, kamel Belloulata "An image segmentation in image processing based on threshold segmentation", Third international IEEE conference on signal-Image Technologies and internetbased system, SITIS 2007 ,pp. 673-678

[7] Kalelia, F.; Aydina, N.; Ertas, G.;Gulcur, H.O.; "An adaptive approach to the segmentation of DCE-MR images of the breast: comparison with classical thresholding algorithms" ,Computational intelligence on image and signal processing. CIISP 2007,IEEE symposium, pp. 375-379

[8] Raman Maini, Dr.Himanshu Aggarwal "Study and comparison of various edge detection techniques", International journal of image processing, vol. 3: issue(1)

[9] Hong Shan Neoh, Asher Hazan chuk "Adaptive edge detection for real-time video processing using FPGAs", 101 innovation drive, San jose, CA

[10] Spreeuwers, L.J.; Van der Heijden , F.; "Evaluation of edge detection using average risk", Pattern recognition , vol. 3 ,Conference on Image ,speech and signal analysis, pp. 771-774, (2002)

[11] Google Images. Retrieved on November 5, 2011 from http://www.google.ca/imghp?hl=en\&tab=wi. 